\title{
I8. COMMISSION DES LONGITUDES PAR TELEGRAPHIE SANS FIL
}

Président: M. Ferrit, Directeur des services de la Télégraphie militaire, Paris. Membres: MM. Alessio, Bardeloni, Bianchi, Bigourdan, Bowyer, Carnera, Carty, Cooke, Esclangon, Fichot, Gautier, Gonnessiat, Hamy, Hasimoto, Lambert, Moreau, Niethammer, Nijland, Nörlund, Nušl, Ribeiro, Sampson, Stewart, Tinoco, Voûte.

Le Président de la Commission mixte Internationale, qui élabora et parvint à réaliser la grande entreprise de l'automne I926, se plaît à féliciter tous les collaborateurs de l'œuvre commune qui a apporté plus de précision, d'homogénéité et de cohésion dans le rattachement en longitude d'un grand nombre de points de notre globe. L'opération qui a débuté le $\mathrm{I}^{\circ}$ Octobre pour prendre fin le $\mathrm{I}^{\circ}$ Décembre avait réuni l'adhésion de trente nations différentes. Cinquante-deux observatoires avaient manifesté l'intention de participer au travail. Les comptes rendus tirés et distribués par les soins du Président de la Commission au fur et à mesure que les premiers renseignements lui parvenaient ont fait connaître l'activité de quarante-cinq stations, indiquant la statistique des signaux reçus quotidiennement et le nombre des séries d'observations astronomiques. Trente-deux de ces stations ont déjà fourni les données qui ont permis au centre de calcul de Paris (M. LAMBERT) de les rattacher à l'une des trois stations fondamentales, ALGER, SAN-DIEGo, ZI-KA-WEI, ainsi qu'il sera indiqué plus loin. Toutefois un certain nombre de participants feront eux-mêmes un exposé détaillé de leurs travaux soit à la Commission, soit dans des publications spéciales. Les nombres définitifs résultant de leur discussion pourront différer très légèrement de ceux que donne le présent résumé, déduit de données souvent succinctes qui ont pu laisser ignorer certains détails, certaines particularités touchant la qualité et l'emploi des observations. Aussi apparaîtra-t-il opportun que le Congrès se prononce sur la publication d'un fascicule d'ensemble réunissant les résultats numériques qu'une entente entre les participants aura jugés les plus sûrs. Il ne faudra pas omettre d'y indiquer d'une façon précise, pour chaque station la situation topographique du point de référence, une simple indication du nom géographique de lieu amenant souvent des confusions.

Les stations fondamentales d'Alger, SAN-Diego, $\mathrm{Zr}$-KA-WEI ont utilisé dans l'ensemble, pour comparer leurs heures locales les signaux émanés de sept postes radiotélégraphiques. La qualité des pendules, leurs comparaisons mutuelles, ainsi que celles faites avec les garde-temps de PARIs, ont montré la légitimité des interpolations. On a pu ainsi utiliser l'ensemble de tous les enregistrements radiotélégraphiques, sans négliger les jours où les observations astronomiques avaient fait défaut. D'ailleurs, le ciel souvent dégagé a permis de nombreuses déterminations astronomiques de l'heure locale ainsi qu'en témoignent les feuilles des comptes rendus.

Un point qu'il y aura lieu de discuter retiendra en particulier l'attention du Congrès, c'est la faible vitesse de propagation des ondes que manifestent les observations. Elles conduisent au chiffre approximatif de $250,000 \mathrm{kilom}$. sec.

Le Bureau de calculs de PARIS a conclu les valeurs suivantes:

$\begin{array}{crrcc} & \text { h. } & \text { m. } & \text { s. } & \text { s. } \\ \text { Différence de longitude: AlGER-SAN-DIEGo } & \mathbf{8} & \mathbf{0} & \mathbf{5 6 . 9 0 0} \pm \mathbf{0 . 0 0 2} \\ \text { SAN-DIEGO-ZI-KA-WEI } & \mathbf{8} & \mathbf{5} & \mathbf{2 8 . 7 3 1} \pm 0.006 \\ \text { ZI-KA-WEI-ALGER } & \mathbf{7} & \mathbf{5 3} & \mathbf{3 4 . 3 6 2} \pm 0.006 \\ \text { Somme... } & \mathbf{2 3} & \mathbf{5 9} & \mathbf{5 9 . 9 9 3} \\ \text { I82 } & & & \end{array}$


Les trente-deux observatoires figurant dans les listes ci-après ont été rattachés à celle des stations fondamentales qui leur est la plus voisine en longitude. Leur rattachement à GREENWICH et à PARIS résulte des éléments suivants obtenus par nos calculs.

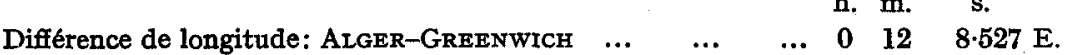

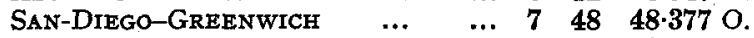

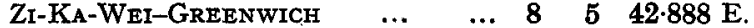

$$
\begin{aligned}
& \text { Paris (Mer, de Cassini)-Greenwich } 0090.913 \mathrm{E} . \\
& \text { G. FERRIE }
\end{aligned}
$$

I5 mai, 1928

Stations Rattachées à Alger

Différence de longitude avec

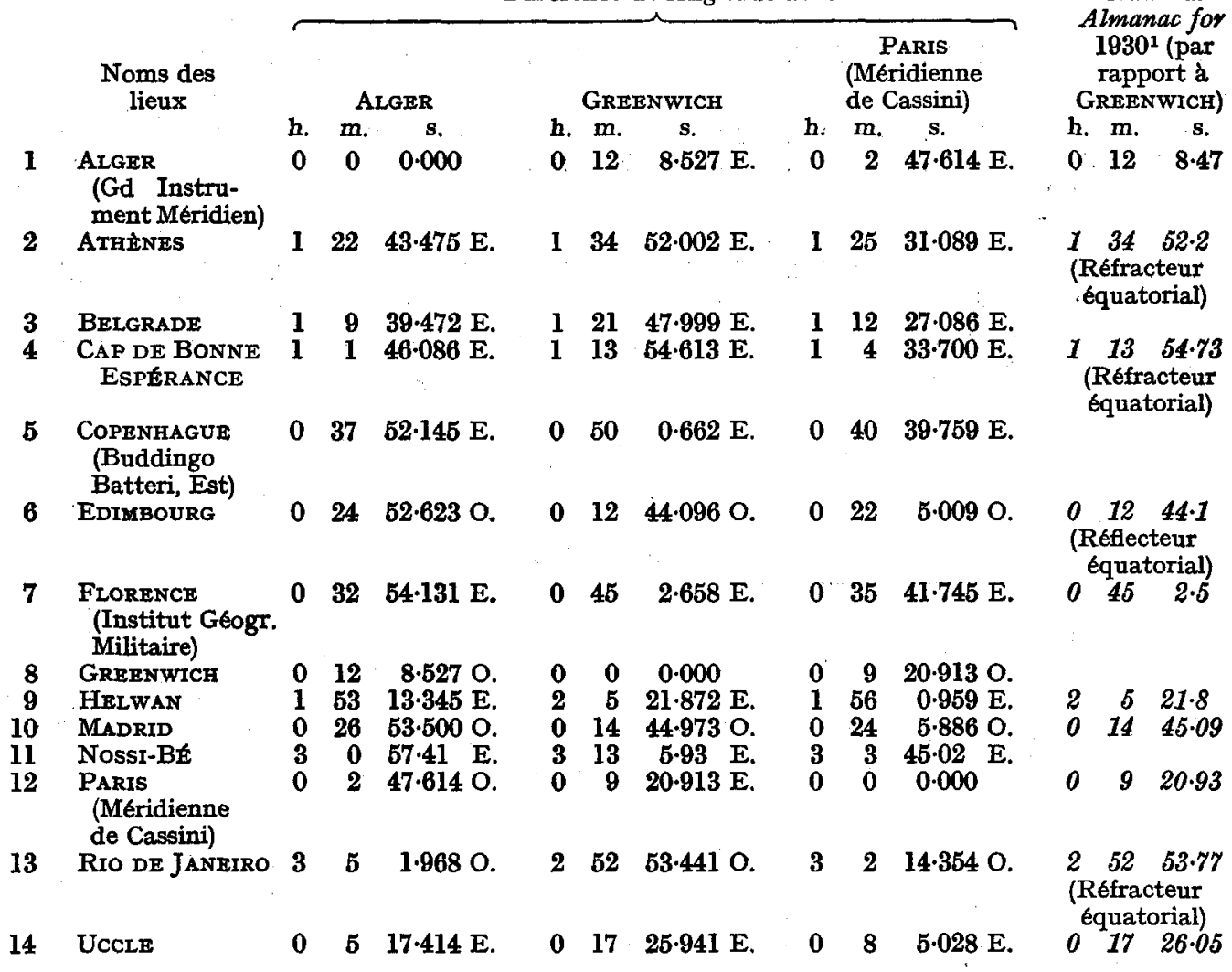

1 D'après les indications de l'Ephéméride. Ces valeurs ont été fournies par chaque Observatoire en réponse à un questionnaire adressé par la Rédaction du Nautical Almanac.
Valeurs extraites du Nartical Almanac for $1930^{1}$ (par rapport à GREENWICH)

b. m. s. $\begin{array}{lll}1 & 34 & 52 \cdot 2\end{array}$ Réfracteur $1354 \cdot 73$ (Refracteur equatorial) Reflecteur équatorial)

$$
\mathbf{I} 83
$$




\section{Statrons RattachéEs a ZI-Ka-WeI}

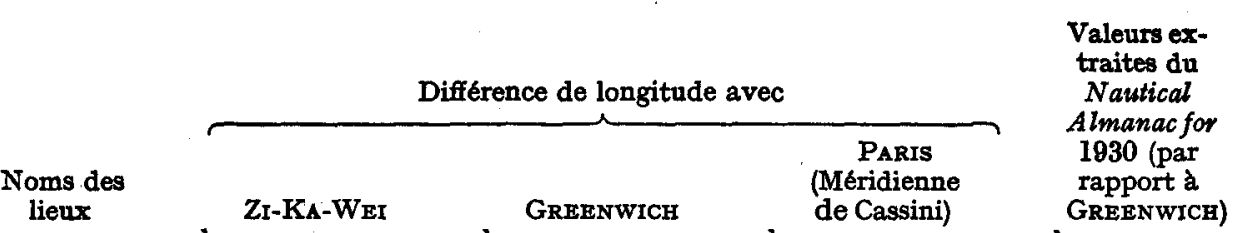

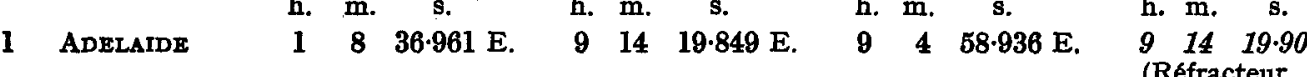

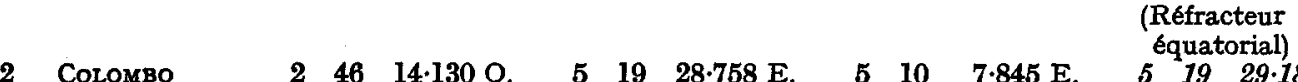

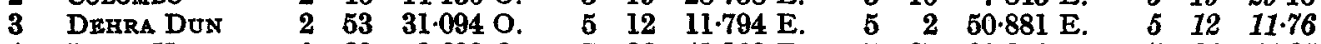

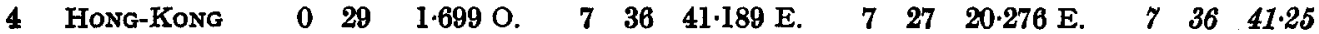

(Cercle Mé-

ridien)

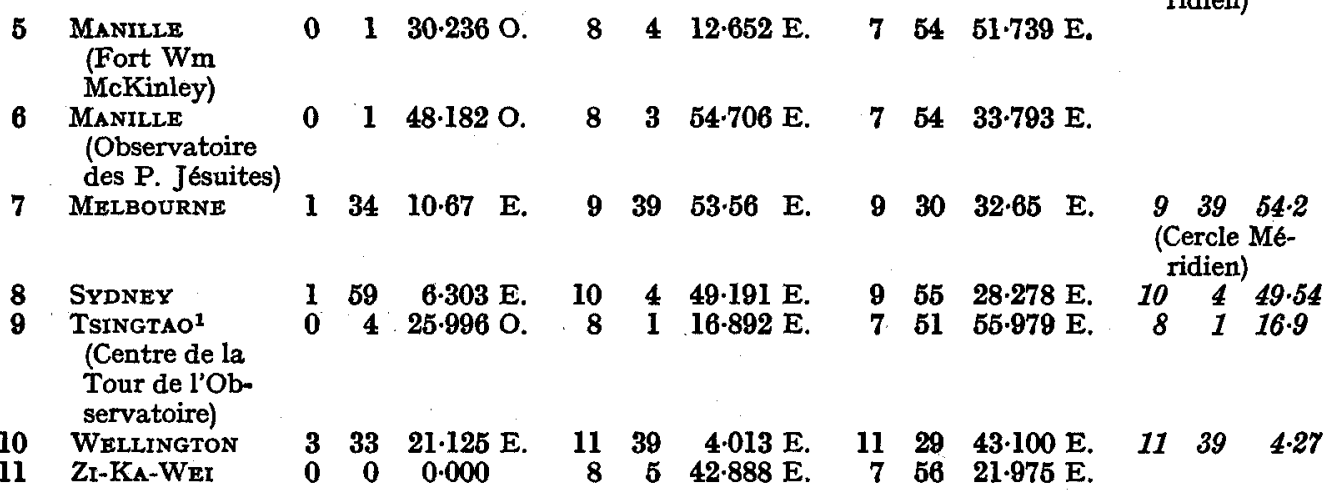

1 Les nombres relatifs à cette station sont déduits de la longitude par rapport à Greenwich $\left(8^{\mathbf{h}} \mathbf{1 m}^{\mathrm{m}}\right.$ 16:892) calculée par l'Observatoire de Tsingtao.

\section{Stations rattachées à San-Diego}

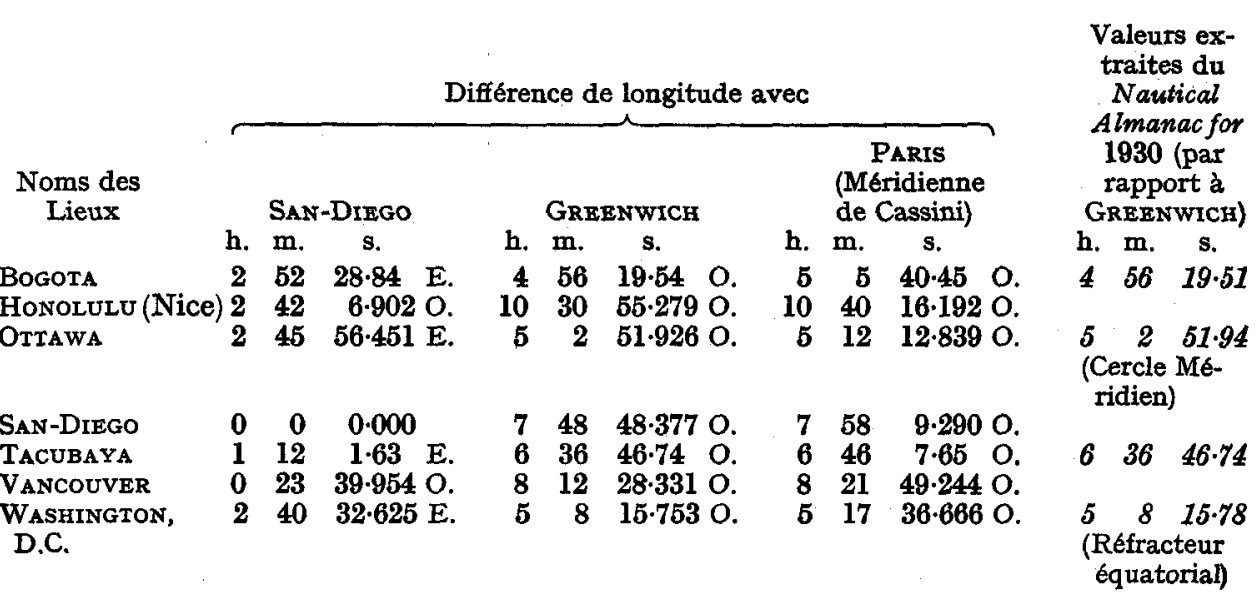

